\title{
MetS Risk Score: A Clear Scoring Model to Predict a 3-Year Risk for Metabolic Syndrome
}

Authors

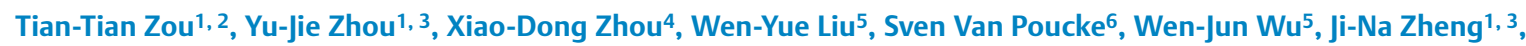
Xue-Mei Gu${ }^{5}$, Dong-Chu Zhang7 ${ }^{7}$ Ming-Hua Zheng', 8 , Xiao-Yan Pan ${ }^{5}$

Affiliations

1 Department of Hepatology, Liver Research Center, The First Affiliated Hospital of Wenzhou Medical University, Wenzhou, China

2 School of the Second Clinical Medical Sciences, Wenzhou Medical University, Wenzhou, China

3 School of the First Clinical Medical Sciences, Wenzhou Medical University, Wenzhou, China

4 Department of Cardiovascular Medicine, The First Affiliated Hospital of Wenzhou Medical University, Wenzhou, China

5 Department of Endocrinology, The First Affiliated Hospital of Wenzhou Medical University, Wenzhou, China

6 Department of Anesthesiology, Ziekenhuis Oost-Limburg, Genk, Belgium

7 Wenzhou Medical Center, Wenzhou People's Hospital, Wenzhou, China

8 Institute of Hepatology, Wenzhou Medical University, Wenzhou, China

\section{Key words}

metabolic syndrome, prediction model, risk factors, general population

received $\quad 13.02 .2018$

accepted $\quad 06.08 .2018$

Bibliography

DOI https://doi.org/10.1055/a-0677-2720

Horm Metab Res 2018; 50: 683-689

(c) Georg Thieme Verlag KG Stuttgart · New York

ISSN 0018-5043

Correspondence

Ming-Hua Zheng, MD, PhD

Department of Hepatology

Liver Research Center

The First Affiliated Hospital of Wenzhou Medical University Institute of Hepatology

Wenzhou Medical University

No. 2 Fuxue Lane

325000 Wenzhou

China
Tel.: + 86/577/88078 232, Fax: + 86/577/88078 262

blueman1320@163.com

Xiao-Yan Pan, MD

Department of Endocrinology

The First Affiliated Hospital of Wenzhou Medical University

No. 2 Fuxue Lane

325000 Wenzhou

China

Tel.: + 86/138/57735 773, Fax: + 86/577/55579 329

xiaoyanpan@wmu.edu.cn

$\Theta$

Supplementary material for this article is available online at http://www.thieme-connect.de/products

\section{ABSTRACT}

Although several risk factors for metabolic syndrome (MetS) have been reported, there are few clinical scores that predict its incidence. Therefore, we created and validated a risk score for prediction of 3-year risk for MetS. Three-year follow-up data of 4395 initially MetS-free subjects, enrolled for an annual physical examination from Wenzhou Medical Center were analyzed. Subjects at enrollment were randomly divided into the training and the validation cohort. Univariate and multivariate logistic regression models were employed for model development. The selected variables were assigned an integer or half-integer risk score proportional to the estimated coefficient from the logistic model. Risk scores were tested in a validation cohort. The predictive performance of the model was tested by computing the area under the receiver operating characteristic curve (AUROC). Four independent predictors were chosen to construct the MetS risk score, including BMI (HR $=1.906$, $95 \% \mathrm{Cl}: 1.040-1.155)$, FPG (HR=1.507, $95 \% \mathrm{Cl}: 1.305-1.741)$, DBP $(H R=1.061,95 \% \mathrm{Cl}: 1.002-1.031), \mathrm{HDL}-\mathrm{C}(\mathrm{HR}=0.539$, $95 \% \mathrm{Cl}: 0.303-0.959)$. The model was created as -1.5 to 4 points, which demonstrated a considerable discrimination both in the training cohort $(A U R O C=0.674)$ and validation cohort (AUROC $=0.690)$. Comparison of the observed with the estimated incidence of MetS revealed satisfactory precision. We developed and validated the MetS risk score with 4 risk factors to predict 3-year risk of MetS, useful for assessing the individual risk for MetS in medical practice. 


\section{Abbreviations}

$\begin{array}{ll}\text { ALB } & \text { Albumin } \\ \text { ALP } & \text { Alkaline phosphatase } \\ \text { ALT } & \text { Alanine aminotransferase } \\ \text { AST } & \text { Aspartate aminotransferase } \\ \text { AUROC } & \text { The area under the receiver operating characteristic curve } \\ \text { BMI } & \text { Body mass index } \\ \text { Cl } & \text { Confidence interval } \\ \text { Cr } & \text { Creatinine } \\ \text { DBP } & \text { Diastolic blood pressure } \\ \text { FPG } & \text { Fasting plasma glucose } \\ \text { GGT } & \text { Y-Glutamyl transferase } \\ \text { HDL-C } & \text { High-density lipoprotein cholesterol } \\ \text { HR } & \text { Hazard ratio } \\ \text { LDL-C } & \text { Low-density lipoprotein cholesterol } \\ \text { MetS } & \text { Metabolic syndrome } \\ \text { NAFL } & \text { Non-alcoholic fatty liver } \\ \text { PLT } & \text { Platelet count } \\ \text { ROC } & \text { Receiver operator characteristic } \\ \text { SBP } & \text { Systolic blood pressure } \\ \text { STB } & \text { Serum total bilirubin } \\ \text { TC } & \text { Total cholesterol } \\ \text { TC } & \text { Triglyceride } \\ \text { UA } & \text { Uric acid } \\ \text { WBC } & \text { White blood cell count } \\ & \end{array}$

\section{Introduction}

Metabolic syndrome (MetS), characterized by a group of metabolic disturbances including central obesity, glucose intolerance, hypertension, and hyperlipidemia [1] is a growing public health problem worldwide [2] with an increasing prevalence [3]. Additionally, MetS is associated with the risk of diabetes, cardiovascular disease, and even death [4-6]. Therefore, more attention should be paid to the prevention of MetS. A prediction model, which is able to identify the individuals with higher risk of MetS development, is urgently needed to estimate the risk of MetS leading to proper interventions at an earlier stage.

Previous studies have reported many related risk factors associated with the incidence of MetS [7-9], such as uric acid (UA), $\mathrm{Y}$-glutamyl transpeptidase (GGT), and alanine aminotransferase (ALT). However, there are few studies grouping these factors to develop a model and predict the risk of MetS. Only one study [10] published in 2015 has identified several independent risks and created a composite score to predict the incidence of MetS. Based on a Japanese employees database, a model was developed to discriminate MetS from healthy individuals and evaluated the predictive potential for recovery from MetS. However, the model contains nine variables including five used to diagnose MetS plus four independent factors, limiting its application.

In this study, we identified four items related to the MetS, constructed and validated a clear model based on routine laboratory and anthropometric parameters to predict a 3-year risk of MetS.

\section{Subjects and Methods}

\section{Study population}

In this study, we screened 4395 initially MetS-free patients who underwent an annual health examination at Wenzhou Medical Center of Wenzhou People's Hospital from 2010 to 2014. The examination includes anthropometric measurements, blood tests, and a physical examination. The entire database was then randomly divided in a 2:1 ratio into training ( 1365 males and 1565 females, age $41.75 \pm 14.70$ years) and validation cohort (681 males and $781 \mathrm{fe}-$ males, age $41.95 \pm 14.89$ years). In addition, verbal informed consent was obtained from each participant and the protocol of the study was approved by the ethics committee of the Wenzhou People's Hospital, respectively.

\section{Event criteria}

MetS was defined according to the guidelines as proposed by the China Diabetes Federation [11] , which indicates that MetS is present if at least three of the following parameters are present: 1) Central obesity: $\mathrm{BMI} \geq 25$ in both genders; 2) Hypertriglyceridemia: TG $\geq 1.7 \mathrm{mmol} / \mathrm{l} ; \mathrm{HDL}-\mathrm{C}<0.9 \mathrm{mmol} / \mathrm{l}$ in males and $<1.0 \mathrm{mmol} / \mathrm{l}$ in females; 3) Hypertension: SBP $\geq 140 \mathrm{mmHg}$ or DBP $\geq 90 \mathrm{mmHg}$ or previously diagnosed; and 4) Hyperglycemia: $F P G \geq 6.1 \mathrm{mmol} / \mathrm{l}$, or hyperglycemia previously diagnosed.

\section{Data collection}

All subjects were instructed to refrain from exercise prior to their examination with clinical examination and data recording conducted the next morning. Medical history, lifestyle, and drug regimes were recorded by well-trained medical staff. Anthropometric measurements included body height and weight. The body mass index (BMI), was calculated by dividing weight $(\mathrm{kg})$ by the square of height $(\mathrm{m})$. Blood pressure, including systolic blood pressure (SBP) and diabolic blood pressure (DBP), was measured automatically (OMRON, Japan), lege artis. Fasting blood samples were collected from each subject and were used for the analysis of biochemical laboratory test. The laboratories were certified according to International Organization Standardization. Laboratory parameters included albumin, white blood cell counts (WBC), blood urea nitrogen (BUN), triglyceride (TG), total cholesterol (TC), low density lipoprotein-cholesterol (LDL-C), high density lipoprotein-cholesterol (HDL-C), serum total bilirubin (STB), fasting plasma glucose (FPG), serum creatinine ( $\mathrm{sCr}$ ), serum uric acid (sUA), alkaline phosphatase (ALP), aspartate aminotransferase (AST), alanine aminotransferase (ALT), and y-glutamyl transferase (GGT). All values were subsequently analyzed by an automated analyzer (Abbott AxSYM, Park, IL, USA) using standard methods.

\section{Statistical analysis}

Continuous variables were summarized as mean \pm standard deviation (SD), and categorical variables were expressed as percentages (\%). The characteristics of the study population according to database were assessed using one way analysis of variance (ANOVA) and $\mathrm{X}^{2}$ test for categorical variables. Univariate and multivariate logistic regression analyses were used to determine the risk factors for MetS. Additionally, a stepwise multivariable logistic regression model was employed to develop a predictive model from the training cohort. For all analysis, two-tailed $p$-value $<0.05$ were consid- 
ered statistically significant and a p-value $<0.1$ was considered indicative of a statistical trend. Data analyses were conducted using SPSS statistics software (version 22; IBM Corp.) and MedCalc version 12.7 (MedCalc Software).

\section{Variable selection}

First, several potential MetS risk factors based on recent literature were selected for evaluation. Univariate association analysis including potential risk factors was conducted and variables with $\mathrm{p}<0.05$ were considered significant. Finally we retained, the four variables that performed well both in the univariate and multivariate analysis for the final model.

\section{Construction of the MetS risk score}

For the training cohort, we translated continuous risk factor variables into categorized variables first, and then performed stepwise multivariable logistic regression analysis to compute $\beta$-coefficients for the four variables. For the analysis, the conditional probabilities used for the entry and removal of a factor were 0.05 and 0.10 , respectively. Then, we established a scoring system that assigned risk scores to each variable based on the magnitude of its $\beta$-coefficient in the multivariable logistic regression model. A sum score, which was named the MetS risk score, was calculated for each participant by adding the scores for four variables together. The mean 3-year risk of all possible combinations of risk factors for a specific total score was computed to obtain 3-year risk values ( $\mathbf{F i g . ~ 2 ) . ~ T o ~ a s - ~}$ sess the predictive potential of the model to discriminate "events" from "nonevents", the area under the receiver-operating characteristic curve (AUROC) was calculated.

\section{Validation of the MetS risk score}

The performance of the risk score was evaluated in the validation cohort and entire sample. The predictive performance of the MetS risk score was evaluated using the AUROCs.

\section{Results}

\section{Baseline characteristics of cohort population}

A total of 10419 individuals were initially recruited into the study. Only 4395 (2930 in training cohort and 1465 in validation cohort) individuals were enrolled according to exclusion criteria ( $\triangleright$ Fig. 1). Baseline clinical and biochemical parameters of training and validation cohort are summarized in Table 1S. No significant difference was found between the training $(n=2930)$ and the validation cohorts $(n=1465)$.

Table 2S shows that patients who developed MetS in 3 years had an older age (44.28 vs. 41.52, $\mathrm{p}=0.006)$, a higher BMI $(22.98$ vs. 21.97, $p<0.001)$, FPG (5.60 vs. 5.19, $\mathrm{p}<0.001)$, SBP (123.74 vs. 120.22, $\mathrm{p}=0.001)$, DBP (74.32 vs. 77.03, $\mathrm{p}<0.001), \mathrm{UA}$ (293.74 vs.

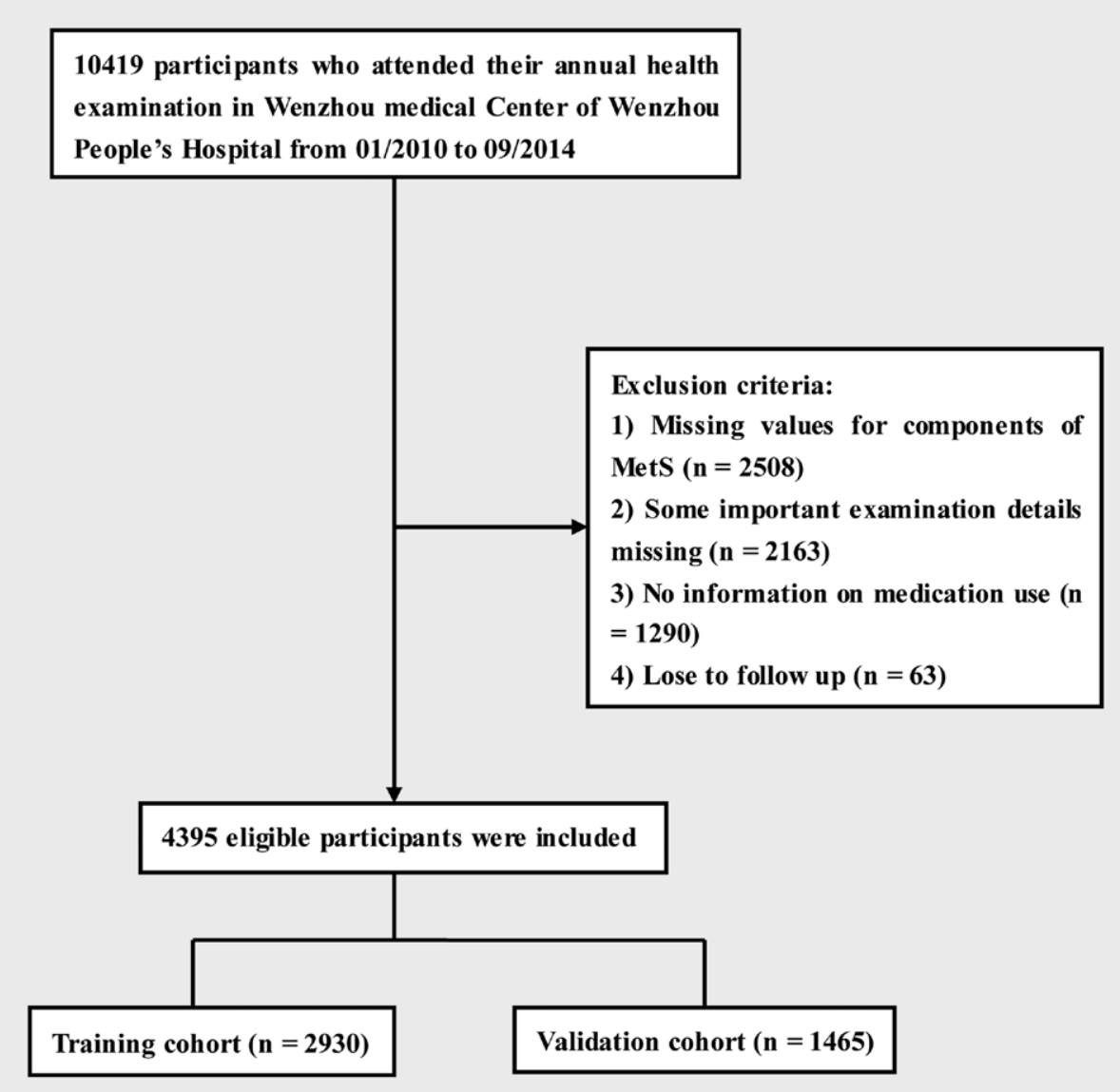

- Fig. 1 Study flow diagram. 
Step1. Assign a score

\begin{tabular}{|cc|}
\hline BMI & Score \\
\hline$<24$ & 0 \\
$\geq 24$ & 1.5 \\
\hline
\end{tabular}

\begin{tabular}{|cc|}
\hline FPG & Score \\
\hline$<5.2$ & 0 \\
$\geq 5.2$ & 1.5 \\
\hline
\end{tabular}

\begin{tabular}{|cc|}
\hline HDL & Score \\
\hline$<1.4$ & 0 \\
$\geq 1.4$ & -1.5 \\
\hline
\end{tabular}

\begin{tabular}{|cc|}
\hline DBP & Score \\
\hline$<73.5$ & 0 \\
$\geq 73.5$ & 1 \\
\hline
\end{tabular}

Step2. Add scores together

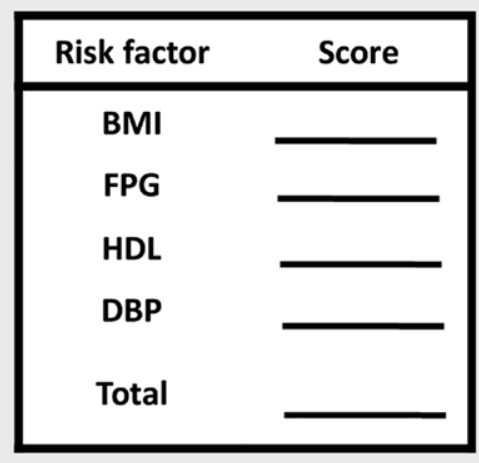

Step3. Find absolute risk

\begin{tabular}{|cc|}
\hline Total Score & $\begin{array}{c}\text { 3-year } \\
\text { risk(\%) }\end{array}$ \\
\hline-1.5 & 0.0 \\
-0.5 & 0.9 \\
0 & 4.8 \\
1 & 6.6 \\
1.5 & 9.9 \\
2.5 & 12.6 \\
3 & 18.4 \\
$\geq 4$ & $\geq 24.1$ \\
\hline
\end{tabular}

Fig. 2 Score sheet for estimating 3-year risk of MetS incidence.

278.49, $\mathrm{p}=0.029)$, GGT (32.72 vs. $25.66, \mathrm{p}<0.001)$, and lower HDL-C ( 1.31 vs. $1.38, \mathrm{p}<0.001)$ in the training cohort while LDL-C, TC and $\mathrm{Cr}$ showed no significant difference. The patients' conditions were similar to the validation cohort.

\section{Development of the MetS risk score}

To identify independent predictors of MetS, the univariate logistic regression analysis was performed to test the relationships between the potential risk factors and the incidence of MetS in the training cohort (Table 3S). In the univariate analysis, we found that age, BMI, SBP, DBP, TG, TC, FPG, GGT, HDL-C, UA, and WBC were significantly associated with MetS development (all $p<0.05$ ).

The above variables that were significantly associated with the risk of MetS were consequently entered into the multivariable logistic regression analysis to select independent predictors. Finally, as presented in $>$ Table 1, BMI (HR=0.091, 95\% Cl: 1.040-1.155), FPG $(\mathrm{HR}=1.507,95 \% \mathrm{Cl}: 1.305-1.714, \mathrm{p}<0.001), \mathrm{DBP}(\mathrm{HR}=0.016,95 \%$ Cl: 1.002-1.031), HDL (HR=0.539, $95 \% \mathrm{Cl}: 0.303-0.959)$, were identified as the independent risk factors.

- Table 2 illustrates the results of the multivariate logistic regression performed on the 4 variables and the method to calculate the MetS score. For these 4 variables, the cut-off value of each parameter to distinguish two severity categories with maximum
- Table 1 Multivariate analysis of the association between incidence and clinical and biochemical characteristics in the training cohort.

\begin{tabular}{|l|c|c|c|r|}
\hline Variables & B & HR & $\mathbf{9 5 \%} \mathbf{C l}$ & p-Value \\
\hline BMI & 0.091 & 1.096 & $1.040-1.155$ & 0.001 \\
\hline FPG & 0.410 & 1.507 & $1.305-1.741$ & $<0.001$ \\
\hline DBP & 0.016 & 1.016 & $1.002-1.031$ & 0.021 \\
\hline HDL-C & -0.617 & 0.539 & $0.303-0.959$ & 0.035 \\
\hline
\end{tabular}

B: Intercept. For abbreviations, see text.

Youden Index in order to predict the risk of MetS was calculated. Then we derived an integer or half-integer score for prediction based on the multivariable logistic regression coefficients. The lowest value of $\beta$-coefficients was chosen to obtain a score of 1 . Other $\beta$-coefficient values were then divided to the lowest value and resulted as score for each variable. Each point was rounded to an integer and half-integer. As a result, we assigned 1.5 points to the predictor BMI $\left(\mathrm{kg} / \mathrm{m}^{2}\right) \geq 24$ and FPG $(\mathrm{mmol} / \mathrm{l}) \geq 5.2,-1.5$ points to the predictor $\mathrm{HDL}-\mathrm{C}(\mathrm{mmol} / \mathrm{I}) \geq 1.4$ and 1 point to the predictor 
> Table 2 MetS risk score based on multivariable analysis of risk factors for MetS in the training cohort.

\begin{tabular}{|c|c|c|c|c|c|}
\hline Categorical variable & Range & $\boldsymbol{\beta}$ & p-Value & HR (95\% Cl) & Risk score \\
\hline \multirow[t]{2}{*}{ BMI $\left(\mathrm{kg} / \mathrm{m}^{2}\right)$} & $<24$ & Reference & - & 1.00 & 0 \\
\hline & $\geq 24$ & 0.790 & $<0.001$ & $2.204(1.661-2.924)$ & 1.5 \\
\hline \multirow[t]{2}{*}{$\mathrm{FPG}(\mathrm{mmol} / \mathrm{I})$} & $<5.2$ & Reference & - & 1.00 & 0 \\
\hline & $\geq 5.2$ & 0.695 & $<0.001$ & $2.004(1.524-2.637)$ & 1.5 \\
\hline \multirow[t]{2}{*}{ HDL-C (mmol/I) } & $<1.4$ & Reference & - & 1.00 & 0 \\
\hline & $\geq 1.4$ & -0.780 & $<0.001$ & $0.458(0.312-0.612)$ & -1.5 \\
\hline \multirow[t]{2}{*}{$\mathrm{DBP}(\mathrm{mmHg})$} & $<73.5$ & Reference & - & 1.00 & 0 \\
\hline & $\geq 73.5$ & 0.467 & 0.001 & $1.595(1.199-2.123)$ & 1 \\
\hline
\end{tabular}
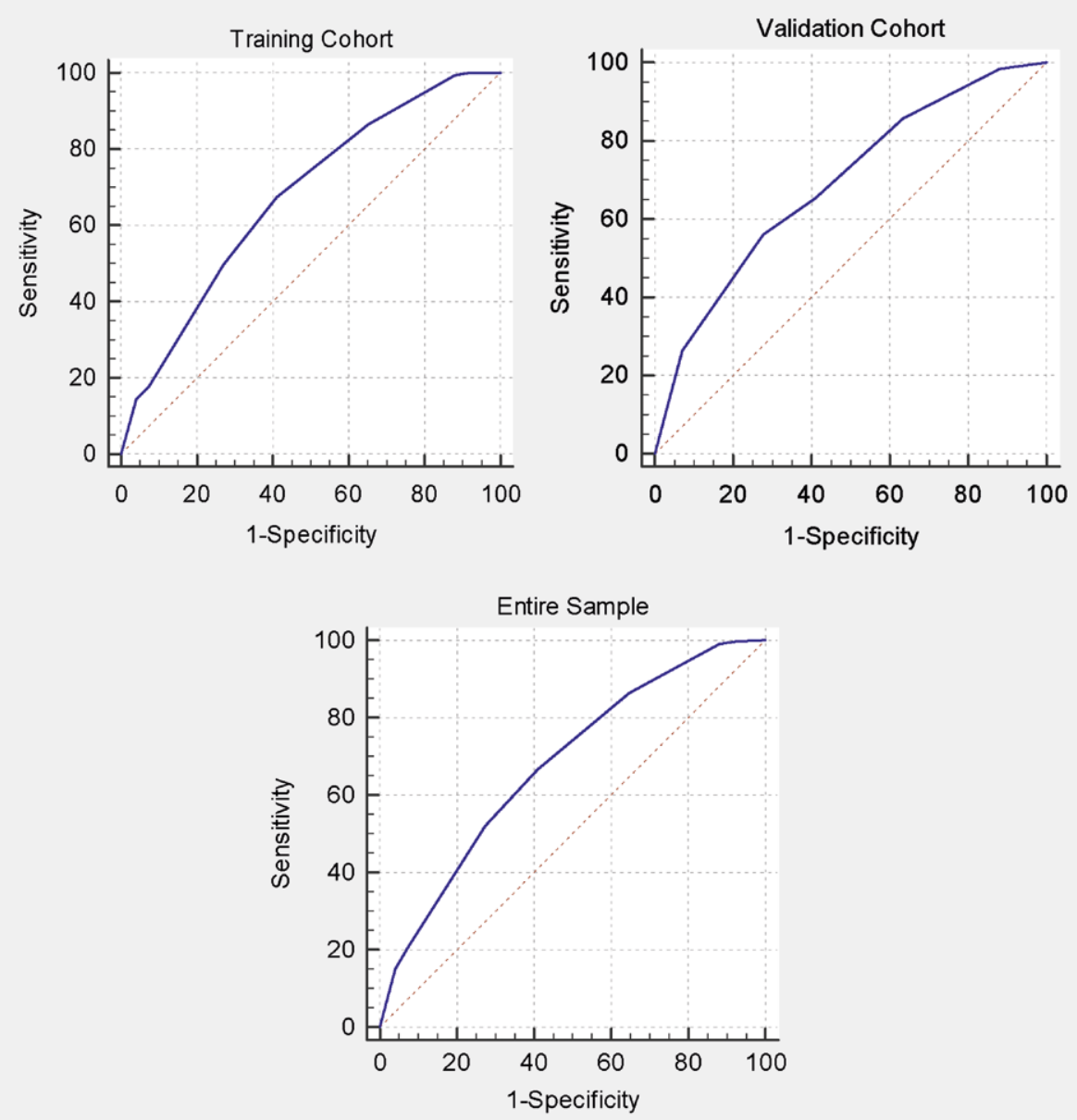

- Fig. 3 Receiver operating characteristic curves analysis of the discriminative ability of MetS risk score to predict 3-year MetS development risk in the general population.

$\operatorname{DBP}(\mathrm{mmHg}) \geq 73.5$. The final score per subject ranged from -1.5 to 4 . A risk estimation chart based on combinations of different points of the four predictors in the MetS risk score was drawn for individual risk prediction ( $\mathbf{F i g} \mathbf{4}$ ).

\section{Performance of MetS score in the training cohort}

- Fig. 3 illustrates the ROC curves in the training cohort, validation cohort and entire sample. The area under the receiver operating characteristic curve (AUROC) was 0.674 for training cohort, 0.690 for validation cohort and 0.680 for the entire database, implying that the discrimination of the model was good. Observed and pre- 


\begin{tabular}{|c|c|c|c|c|c|c|}
\hline \multicolumn{2}{|c|}{ BMI } & $<24$ & $\geq 24$ & $<2$ & $\geq 24$ \\
\hline \multirow{2}{*}{ FPG } & HDL-C & \multicolumn{2}{|c|}{ DBP $<73.5$} & \multicolumn{2}{c|}{ DBP $\geq 73.5$} \\
\hline \multirow{2}{*}{$<5.2$} & $<1.4$ & 6.1 & 10.1 & 6.6 & 13.6 \\
\cline { 2 - 7 } & $\geq 1.4$ & 0.0 & 0.0 & 0.9 & 6.3 \\
\hline \multirow{2}{*}{$\geq 5.2$} & $<1.4$ & 10.0 & 8.1 & 9.9 & 24.0 \\
\cline { 2 - 7 } & $\geq 1.4$ & 1.3 & 15.0 & 6.7 & 25.0 \\
\hline
\end{tabular}

- Fig. 4 Risk estimation chart of MetS risk score.

dicted ratios of 3-year incidence/risk (\%) of MetS was detailed in Fig. 1S, which was calculated according to risk values revealed in - Fig. 2, suggesting no significant difference between observed and predicted ratios in both training cohort ( $8.98 \%$ vs. $8.16 \%$ ) and validation cohort ( $8.66 \%$ vs. $8.73 \%$ ).

\section{Discussion}

In this study, we established and validated a new MetS risk score to predict the risk of MetS within the next three years. To our knowledge, this is the first study that developed a prediction score for incident MetS using the components of MetS. According to the result of the AUROC, the model showed a good predictability. Physicians can employ the MetS Risk Score to make individual predictions easily, and identify those potential patients and inform them to promote healthier behaviors to prevent the disease.

As we know, there are many diagnostic criteria of MetS around the world [11-13]. such as the criteria from the Joint Interim Statement of the International Diabetes Federation Task Force of Epidemiology and Prevention American Heart Association, World Heart Federation, International Atherosclerosis Society, American Heart Association, International Association for the Study of Obesity and China Diabetes Federation. Finally, considering the composition of the population and the popularity of the criteria, MetS was diagnosed according to the China Diabetes Federation, which was composed of Central Obesity, Hypertriglyceridemia, Hypertension and Hyperglycemia.

Several risk factors of MetS have been identified in our study. First, we found that abdominal obesity $(\mathrm{BMI})(\beta=0.790, \mathrm{HR}=2.204)$ was the most important contributor to the incidence of MetS among the four items included in the score, which is in line with others [14] indicating that intra-abdominal fat is a major determinant for the metabolic syndrome. Recent researches $[15,16]$ showed that GGT is also associated with the risk of developing MetS, which was also identified in our study. The mechanisms have not been fully elucidated, but a study found that GGT may contribute to incident MetS via inflammation, oxidative stress pathways and insulin resistance [17]. However, due to the poor performance in the multivariable logistic regression analysis, it was not included in the score. Although the effect of gender difference on the incidence of the syndrome remains uncertain, we discovered that female sex could also be regarded as a risk score as a previous study demonstrated that female sex is an independent item to predict MetS in the United States [18]. Therefore, further research is required to validate if female sex can also be regarded as a risk factor in Asian population. Additionally, non-alcoholic fatty liver (NAFL) demonstrated a tight connection with the MetS [19]. Its components have also been reported to independently predict the risk of NAFL in a northern urban Han Chinese population [20]. However, we found no significant association between MetS and NAFL in our study. One possible explanation is that NAFL may gradually disappear in a 3-year follow up not directly predicting the appearance of MetS. Elevated uric acid was also reported to be associated with MetS, which was confirmed in several studies [8,21,22] . The exclusion of this important factor may have influenced the difference of diagnostic criteria. Finally, considering the performance both in multivariate analysis and AUROC, we identified four risk factors, BMI, FPG, HDL-C and DBP in our model.

There are several strengths shown in our study. First, this is the first clean model based on routine laboratory and anthropometric parameters to predict a 3-year risk of MetS. The items included in the model are easy to obtain facilitating implementation. Individuals, who were identified at high-risk for MetS, can initiate a healthier lifestyle.

Despite the strengths of the present study, there are some limitations that need to be addressed. First, diagnoses of MetS in our study were based on the China Diabetes Federation, which is not universally implemented but considered most suitable for our study. Second, because of insufficient information retrieval, we could not evaluate lifestyle related parameters such as smoking, drinking and physical activity in this study [23]. Third, related with a limited dataset, the scoring model was not validated in different centers. We randomly divided the data into two parts: one to build the model and another to validate its performance. Validation could be optimized by $\mathrm{X}$-validation. Further research is required to validate the scoring model in other multicenter studies.

\section{Conclusions}

In conclusion, we have developed a clear scoring model, the MetS risk score, for evaluating the 3-year risk of MetS individually. Our model identified 4 predictors to score the risk and performed well both in the training cohort and validation cohort. 


\section{Authors' Contributions}

Tian-Tian Zou: designed the study, did the statistical analyses, interpreted data, and wrote the manuscript. Yu-jie Zhou: did the statistical analyses and collected data. Xiao-Dong Zhou and Wen-Yue Liu: interpreted data and revised the manuscript. Sven Van Poucke: revised the manuscript. Wen-Jun Wu and Dong-Chu Zhang: data collection. Ji-Na Zheng and Xue-Mei Gu: interpreted data. MingHua Zheng and Xiao-Yan Pan: designed the study, obtained funding, reviewed the results, and helped to write the manuscript. All authors saw and approved the final version of the paper.

\section{Funding}

This work was supported by grants from the National Natural Science Foundation of China (81500665), Scientific Research Foundation of Wenzhou (Y20160223), High Level Creative Talents from Department of Public Health in Zhejiang Province, and Project of New Century 551 Talent Nurturing in Wenzhou.

\section{Conflict of Interest}

The authors declare that they have no conflict of interest.

\section{References}

[1] Grundy SM, Brewer HB Jr, Cleeman JI, Smith SC Jr., Lenfant C. Definition of metabolic syndrome: Report of the National Heart, Lung, and Blood Institute/American Heart Association conference on scientific issues related to definition. Arterioscler Thromb Vasc Biol 2004; 24: e13-e18

[2] Ford ES, Giles WH, Dietz WH. Prevalence of the metabolic syndrome among US adults: Findings from the third National Health and Nutrition Examination Survey. JAMA 2002; 287: 356-359

[3] Ford ES, Giles WH, Mokdad AH. Increasing prevalence of the metabolic syndrome among U.S. Adults. Diabetes Care 2004; 27: 2444-2449

[4] Gami AS, Witt B], Howard DE, Erwin PJ, Gami LA, Somers VK, Montori VM. Metabolic syndrome and risk of incident cardiovascular events and death: A systematic review and meta-analysis of longitudinal studies. J Am Coll Cardiol 2007; 49: 403-414

[5] Ford ES. Risks for all-cause mortality, cardiovascular disease, and diabetes associated with the metabolic syndrome: A summary of the evidence. Diabetes Care 2005; 28: 1769-1778

[6] Sung KC, Rhee E], Ryu S, Kim B], Kim BS, Lee WY, Oh KW, Kim YB, Chung PW, Kim H, Byrne CD, Lee KB, Park SW. Increased cardiovascular mortality in subjects with metabolic syndrome is largely attributable to diabetes and hypertension in 159,971 korean adults. J Clin Endocrinol Metab 2015; 100: 2606-2612

[7] Ryu S, Song J, Choi BY, Lee S], Kim WS, Chang Y, Kim DI, Suh BS, Sung $\mathrm{KC}$. Incidence and risk factors for metabolic syndrome in Korean male workers, ages 30 to 39. Ann Epidemiol 2007; 17: 245-252

[8] Sui X, Church TS, Meriwether RA, Lobelo F, Blair SN. Uric acid and the development of metabolic syndrome in women and men. Metabolism 2008; 57: 845-852
[9] Zhong P, Sun DM, Wu DH, Li TM, Liu XY, Liu HY. Serum total bilirubin levels are negatively correlated with metabolic syndrome in aged Chinese women: A community-based study. Braz J Med Biol Res 2017; 50: e5252

[10] Obokata M, Negishi K, Ohyama Y, Okada H, Imai K, Kurabayashi M. A risk score with additional four independent factors to predict the incidence and recovery from metabolic syndrome: Development and Validation in Large Japanese cohorts. PLoS One 2015; 10: e0133884

[11] Lu YH, Lu JM, Wang SY, Li CL, Liu LS, Zheng RP, Tian H, Wang XL, Yang LJ, Zhang YQ, Pan CY. Comparison of the diagnostic criteria of metabolic syndrome by International Diabetes Federation and that by Chinese Medical Association Diabetes Branch. Zhonghua Yi Xue Za Zhi 2006; 86: 386-389

[12] Grundy SM, Cleeman JI, Daniels SR, Donato KA, Eckel RH, Franklin BA, Gordon DJ, Krauss RM, Savage PJ, Smith SC Jr., Spertus JA, Costa F. Diagnosis and management of the metabolic syndrome: An American Heart Association/National Heart, Lung, and Blood Institute Scientific Statement. Circulation 2005; 112: 2735-2752

[13] Doi Y, Ninomiya T, Hata J, Yonemoto K, Arima H, Kubo M, Tanizaki Y, Iwase M, lida M, Kiyohara Y. Proposed criteria for metabolic syndrome in Japanese based on prospective evidence: The Hisayama study. Stroke 2009; 40: 1187-1194

[14] Carr DB, Utzschneider KM, Hull RL, Kodama K, Retzlaff BM, Brunzell JD, Shofer JB, Fish BE, Knopp RH, Kahn SE. Intra-abdominal fat is a major determinant of the National Cholesterol Education Program Adult Treatment Panel III criteria for the metabolic syndrome. Diabetes 2004; 53: 2087-2094

[15] Kunutsor SK, Apekey TA, Seddoh D. Gamma glutamyltransferase and metabolic syndrome risk: A systematic review and dose-response meta-analysis. Int J Clin Pract 2015; 69: 136-144

[16] Lee JH, Um MH, Park YK. The association of metabolic syndrome and Serum gamma-glutamyl transpeptidase: A 4-year cohort study of 3,698 Korean male workers. Clin Nutr Res 2013; 2: 67-75

[17] Kerner A, Avizohar O, Sella R, Bartha P, Zinder O, Markiewicz W, Levy Y, Brook G], Aronson D. Association between elevated liver enzymes and C-reactive protein: Possible hepatic contribution to systemic inflammation in the metabolic syndrome. Arterioscler Thromb Vasc Biol 2005; 25: 193-197

[18] Mozumdar A, Liguori G. Persistent increase of prevalence of metabolic syndrome among U.S. adults: NHANES III to NHANES 1999-2006. Diabetes Care 2011; 34: 216-219

[19] Targher G, Byrne CD. A perspective on metabolic syndrome and nonalcoholic fatty liver disease. Metab Syndr Relat Disord 2015; 13: 235-238

[20] Zhang T, Zhang C, Zhang Y, Tang F, Li H, Zhang Q, Lin H, Wu S, Liu Y, Xue F. Metabolic syndrome and its components as predictors of nonalcoholic fatty liver disease in a northern urban Han Chinese population: A prospective cohort study. Atherosclerosis 2015; 240: 144-148

[21] Onat A, Uyarel H, Hergenc G, Karabulut A, Albayrak S, Sari I, Yazici M, Keles I. Serum uric acid is a determinant of metabolic syndrome in a population-based study. Am J Hypertens 2006; 19: 1055-1062

[22] Choi HK, Ford ES. Prevalence of the metabolic syndrome in individuals with hyperuricemia. Am J Med 2007; 120: 442-447

[23] Tran BT, Jeong BY, Oh JK. The prevalence trend of metabolic syndrome and its components and risk factors in Korean adults: Results from the Korean National Health and Nutrition Examination Survey 2008-2013. BMC Public Health 2017; 17: 71 